\title{
Liquorice Induced Hypertension and Hypokalaemia
}

Dr. Hamza Ali Khan*, Dr. Paul Peter\#, Dr. Giridhar Tarigopula\#, Dr Praveen Partha\#

*Specialist Registrar Department of Diabetes \& Endocrinology Darlington Memorial Hospital Darlington \#Consultant Department of Diabetes and Endocrinology Darlington Memorial Hospital Darlinton

\section{Introduction}

Liquorice (scientific name Glycyrrhiza glabra) is historically used for Gl complaints, now it is primarily used as a flavoring agent in the tobacco, confectionery and to some extent in the pharmaceutical and beverage industries. Excessive intake of liquorice can cause hypokalaemia and hypertension We describe a patient with hypokalaemia caused by long term consumption of liquorice. The case emphasizes the importance of considering a detailed patients' history, which often may lead the treating physician to the correct clinical diagnosis.

\section{Case Presentation}

A 69 years old caucasian lady was referred by General Practioner with low serum potassium of $2.0 \mathrm{mmol} / \mathrm{L}$ and dependent oedema. No history of diarrhoea or vomiting and no diuretics or laxative use. She was an ex smoker stopped when she was a teenager and consumed a bottle of wine per week.

Physical examination showed mild hypertension $173 / 88 \mathrm{mmHg}$, Pulse $85 /$ min regular. Dependent oedema up to the ankles. Rest of the physical examination was normal

\section{Investigations}

-Serum potassium of $2.0 \mathrm{mmol} / \mathrm{L}$, Serum sodium 146

$\mathrm{mmol} / \mathrm{L}$,Urea and creatinine normal

- Normal bone profile.

-Serum Magnesium 0.57mmol/L(Normal 0.7-1)

-Arterial $\mathrm{Ph} 7.50$, Bicarbonate $42 \mathrm{mmol} / \mathrm{L}$ ( metabolic alkalosis)

-TSH 2.25 (Normal)

- Heamoglobin, White cell count and Platelets were Normal

- Serum Renin $<0.2 \mathrm{pmol} / \mathrm{ml} / \mathrm{h}$ and Aldosterone $<70 \mathrm{pmol} / \mathrm{L}$

- over night Dexamethasone suppression test was normal at $24 \mathrm{nmol} / \mathrm{L}$.

- Her ultrasound scan of the kidneys was normal.

.24 hours urinary calcium was normal $2.82 \mathrm{mmol} /$ day

\section{Discussion}

- Glycyrrhetinic acid inhibits the conversion of active cortisol to inactive cortisone in the kidneys by inhibiting $11 \beta$-Hydroxysteroid dehydrogenase as well as competitively inhibitining it ${ }^{1,2}$ Cortisol has intrinsic mineralocorticoid properties thus leading to hypertension, hypokakalamia,low renin and aldosteone levels -It can present with fatigue, muscle cramps, hypokalaemia, myoglobinuria ${ }^{3}$, weakness/paralysis ${ }^{4}$, oedema, dyspnoea or Paresthesias/dysesthesias (e.g., burning sensations of extremities). It is known to cause hypertension and hypertensive encephalopathy ${ }^{5}$ -In our patient ultrasound of the kidneys was normal ruling out renal artery stenosis 6 and normal over night dexamethasone ruled out cushing's syndrome

-Although Liddle's syndrome can present in elderly ${ }^{7}$,

our patient's potassium stabilized after discontinuing liqourice ingestion - Gitelman's and Barter's syndrome patients are usually normotensive, hypokalaemic, hypocalciuric and have metabolic alkalosis and raised plasma renin and aldosterone levels ${ }^{8}$

\section{Progress and Management}

She was seen by the Endocrinology team and her history at that time revealed that she had been taking liquorice in sweets for as long as she remembered. Her previous potassium readings couple of years back showed that she was hypokalaemic. She stopped Liquorice after that admission and was discharged home on oral potassium supplements which she gradually reduced and was not on any potassium supplements for about two months when seen in July 2012. She was normotesive at $120 / 60 \mathrm{mmHg}$ with no dependent oedema.

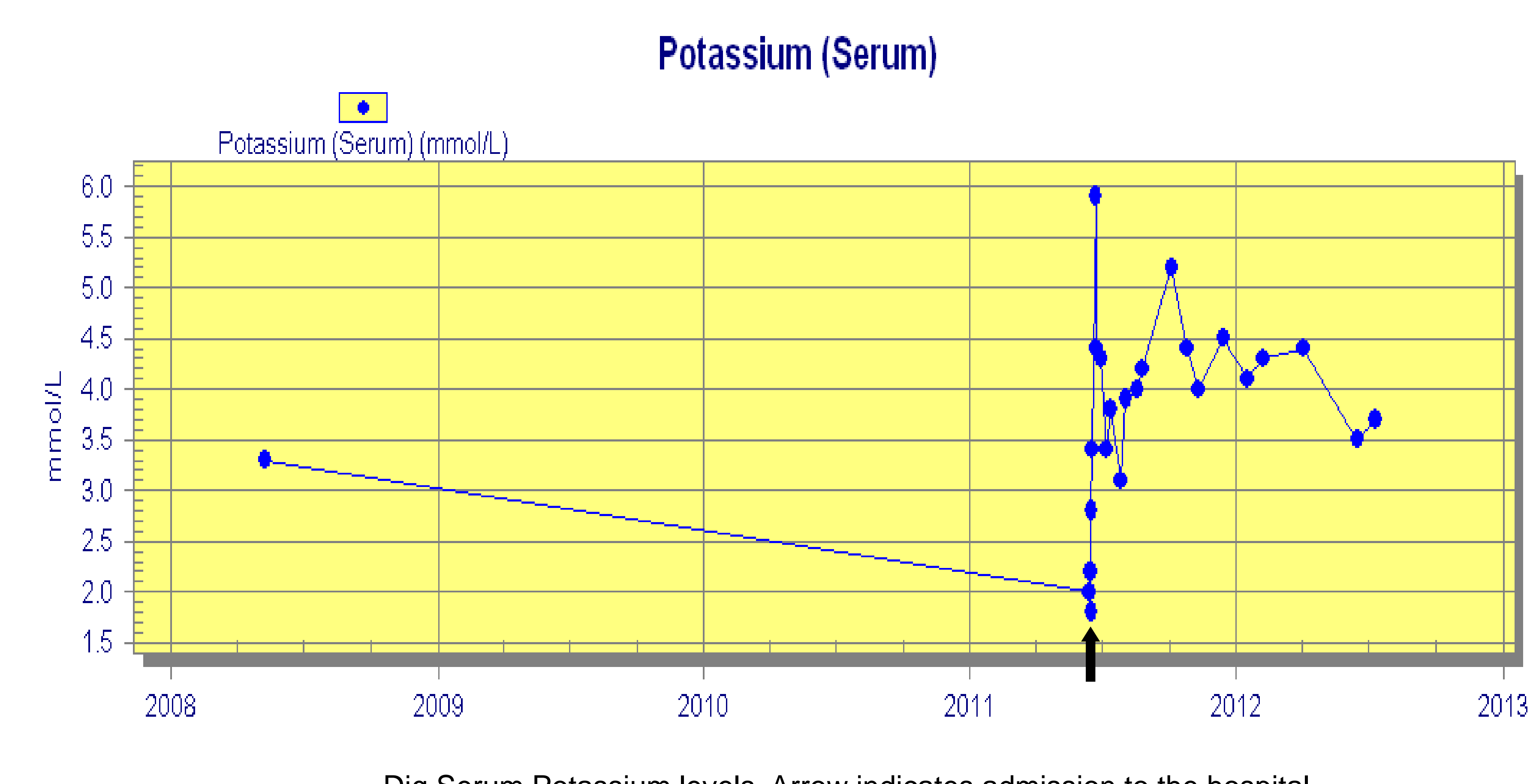

Dig.Serum Potassium levels. Arrow indicates admission to the hospital

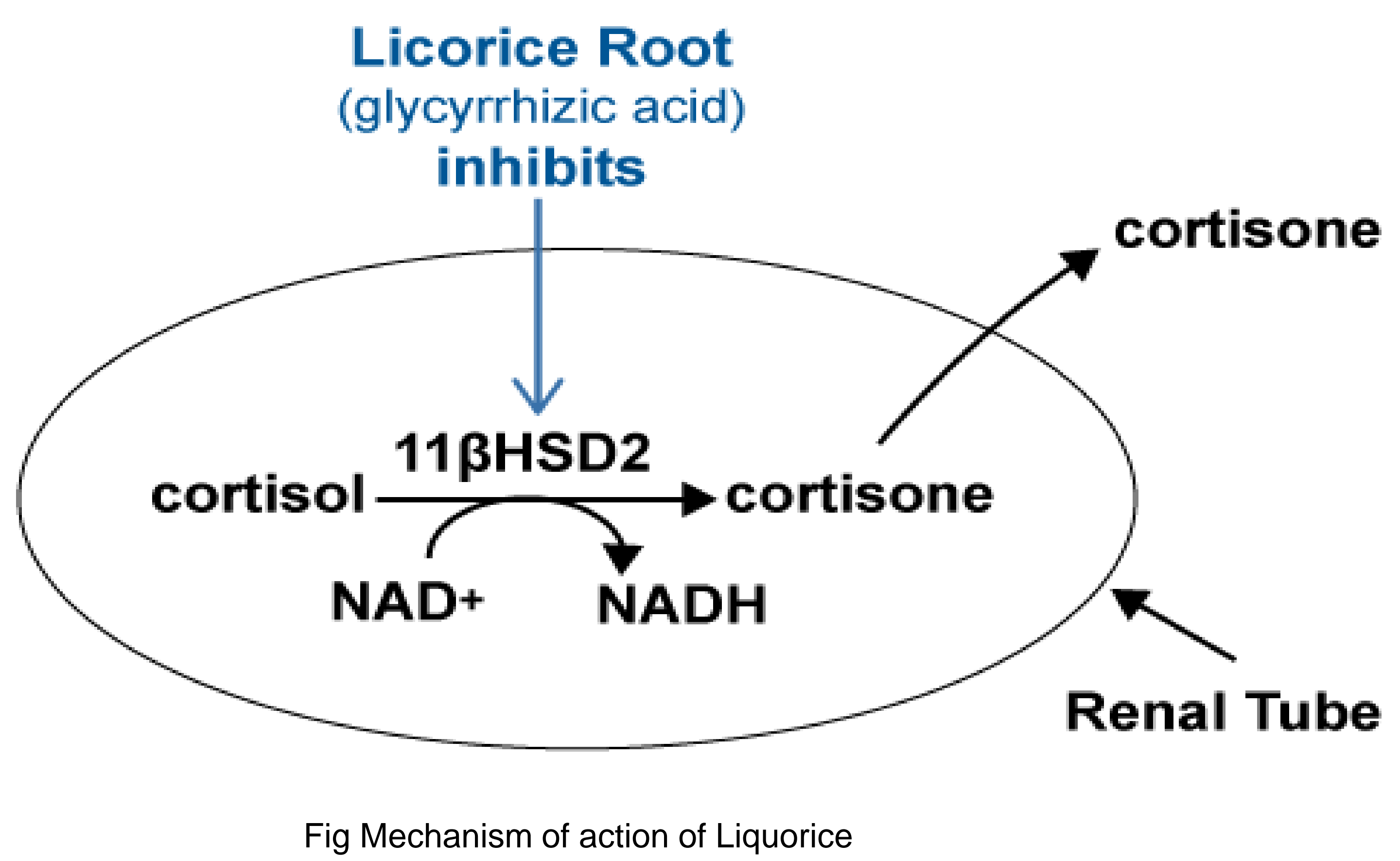

Fig Mechanism of action of Liquorice

\section{Conclusion}

A detailed clinical history is vital to reach clinical diagnosis. In patients, who present with hypokalaemia and hypertension liquorice ingestion should be kept in mind particularly those with low renin and aldosterone levels 